\title{
Cubic Phases and Cubic Phase Dispersions in a Phospholipid Based System
}

\author{
Markus Johnsson ${ }^{\star 1,2}$, Justas Barauskas ${ }^{1,2}$ and Fredrik Tiberg ${ }^{1,2}$ \\ 1. Camurus AB, Ideon, Gamma 2, Sölvegatan 41, SE-223 70, Lund, Sweden \\ 2. Physical Chemistry 1, Center for Chemistry and Chemical Engineering, Lund University, P.O. Box 124, SE-221 00 \\ Lund, Sweden \\ markus.johnsson@camurus.ideon.se
}

\begin{abstract}
Supporting Information
Experimental Details

Materials. Dioleoylphosphatidylethanolamine (DOPE) was obtained from Lipoid $\mathrm{GmbH}$ (Ludwigshafen, Germany). Pegylated glycerol monooleate (PEG(660)-GMO) with an average of 15 ethylene oxide (EO) units was provided by Nikko Chemicals (Tokyo, Japan). The trade name of this surfactant is TMGO-15. The lipid and surfactant were used as received. Sterile water from B. Braun Medical AB (Bromma, Sweden) was used for the preparation of samples.
\end{abstract}

Sample Preparation. Samples for phase behavior studies were prepared by weighing the appropriate amounts of lipid, surfactant and water into small glass vials. The typical sample weight was $0.5 \mathrm{~g}$. The vials were immediately sealed and the components were mixed by vortex mixing and repeated centrifugation. After 2-3 weeks of centrifugation of the samples "up and down" (at least once per day) the samples were put at rest for 1-2 weeks $\left(25^{\circ} \mathrm{C}\right)$ before measurements and phase assignment. The samples were investigated between crossed polarizers to check sample homogeneity and the presence of birefringent phases.

Samples for cryo-TEM investigations were prepared by diluting equilibrated concentrated samples with water and mixing the samples by magnetic stirring (5 days) at $25^{\circ} \mathrm{C}$. The total amphiphile concentration in the cryo-TEM studies was approximately $2 \mathrm{wt} \%$.

X-ray Diffraction (XRD). XRD experiments were performed on a Kratky compact small-angle system equipped with a position-sensitive wire detector (OED 50M from MBraun, Graz, Austria) containing 1024 channels of width $53.6 \mu \mathrm{m} . \mathrm{Cu} \mathrm{K}$ radiation of wavelength $1.542 \AA$ was provided by a Seifert ID $3000 \mathrm{X}$-ray generator operated at $50 \mathrm{kV}$ and $40 \mathrm{~mA}$. The samples were mounted between mica sheets in a steel body. To minimize scattering from air, the camera volume was kept under vacuum during the measurements. Temperature control within $0.1^{\circ} \mathrm{C}$ was achieved using a Peltier element. The recorded slit-smeared diffraction patterns were desmeared and evaluated using 3D-View software (MBraun, Graz, Austria).

Cryo-Transmission Electron Microscopy (cryo-TEM). The samples were prepared in a controlled environment vitrification system. The climate chamber temperature was $25-28^{\circ} \mathrm{C}$ and the relative humidity was kept close to saturation to prevent evaporation from the sample during preparation. A $5 \mu \mathrm{L}$ sample drop was placed on a carbon-coated holey film supported by a copper grid and gently blotted with filter paper to obtain a thin liquid film $(20-500 \mathrm{~nm})$ on the grid. The grid was then rapidly plunged into a liquid ethane at $-180^{\circ} \mathrm{C}$ and transferred into liquid nitrogen $\left(-196^{\circ} \mathrm{C}\right)$. The vitrified specimens were stored in liquid nitrogen and transferred into a Philips CM120 BioTWIN microscope equipped with a post-column energy filter (Gatan GIF 100) using an Oxford CT 3500 cryo-holder and its workstation. The acceleration voltage was $120 \mathrm{kV}$ and the working temperature was kept below $-180^{\circ} \mathrm{C}$. The images were recorded digitally with a CCD camera (Gatan MSC 791) under low-dose conditions with an underfocus of approximately $1 \mu \mathrm{m}$.

\section{Determination of the lattice parameter}

The lattice parameter $(a)$ of the cubic phases was determined by plotting the inverse of the peak spacing $\left(d^{-1}\right)$ versus the square root of the summed squared Miller indices $\left(\mathrm{h}^{2}+\mathrm{k}^{2}+\mathrm{l}^{2}\right)^{1 / 2}$. The slope of the linear fits to the data equals the inverse of the lattice parameter $\left(a^{-1}\right)$. Figure 1_Supporting Information shows the results obtained for the two different cubic phases formed at DOPE/PEG(660)-GMO = 89/11 wt/wt and 45 and $50 \mathrm{wt} \%$ water, respectively.

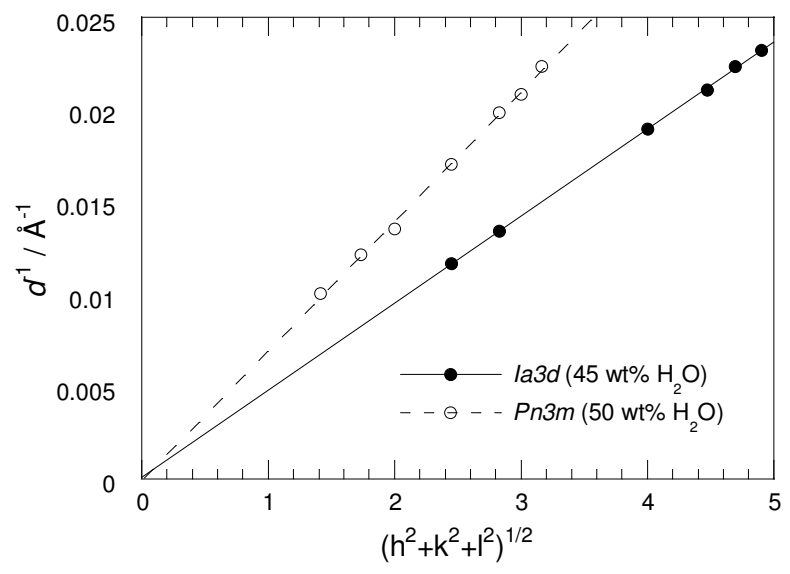

Figure 1_Suporting Information. Inverse of the peak position $d^{-1}$ vs. $\left(\mathrm{h}^{2}+\mathrm{k}^{2}+\mathrm{l}^{1}\right)^{\mathrm{T} / 2}$ for the two cubic lattices shown in Figure 1 of the article. The lines through the data points represent linear fits $\left(\mathrm{R}^{2}=0.99977\right.$ and 0.99786 for the 45 and $50 \mathrm{wt} \% \mathrm{H}_{2} \mathrm{O}$ samples, respectively).

Note that the samples investigated by XRD (Figure 1 of the article and Figure 1_Supporting Information) were macroscopically homogenous and transparent and were regarded as 1-phase samples. 